\title{
Christmas crossword answers
}

\begin{tabular}{|l|l|l|l|l|l|l|l|l|l|l|l|l|l|l|}
\hline R & E & G & R & E & S & S & E & L & E & V & A & T & E \\
\hline E & & A & & M & & W & & C & & S & & B & & N \\
\hline B & E & N & Z & O & D & I & A & Z & E & P & I & N & E & S \\
\hline U & & S & & T & & N & & E & & A & & O & & L \\
\hline F & E & E & L & I & N & G & & M & E & N & S & R & E & A \\
\hline F & & R & & V & & S & E & A & & T & & M & & V \\
\hline S & Y & S & T & E & M & & N & & F & O & L & A & T & E \\
\hline & & S & & & S & U & S & T & O & & & L & & \\
\hline P & S & Y & C & H & E & & U & & B & A & B & B & L & E \\
\hline L & & N & & A & & S & E & C & & L & & E & & I \\
\hline A & D & D & E & N & D & A & & R & U & S & T & L & E & D \\
\hline S & & R & & G & & U & & A & & O & & I & & E \\
\hline M & O & O & D & I & N & C & O & N & G & R & U & E & N & T \\
\hline I & & M & & N & & E & & I & & A & & F & & I \\
\hline D & R & E & D & G & E & R & & A & M & N & E & S & I & C \\
\hline
\end{tabular}

Congratulations to Dr D. W. Forrest, Trinity College, Dublin, who was the crossword winner. 